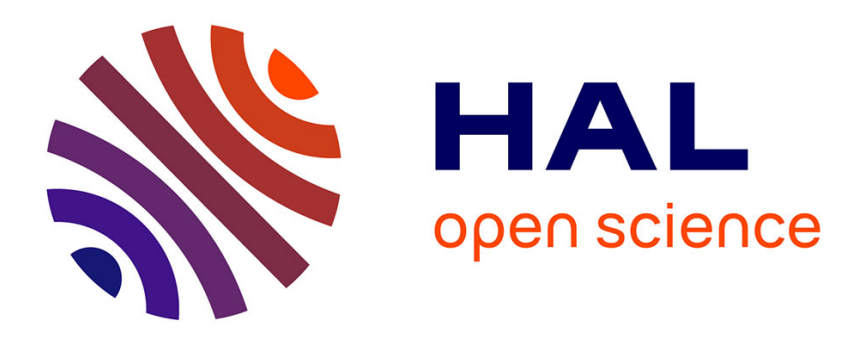

\title{
A thermodynamical approach to contact wear as application of moving discontinuities
}

\author{
Claude Stolz
}

\section{To cite this version:}

Claude Stolz. A thermodynamical approach to contact wear as application of moving discontinuities. Archive of Applied Mechanics, 2007, 77 (2-3), pp.165-175. 10.1007/s00419-006-0057-1 . hal00091378

\section{HAL Id: hal-00091378 \\ https://hal.science/hal-00091378}

Submitted on 18 Jun 2007

HAL is a multi-disciplinary open access archive for the deposit and dissemination of scientific research documents, whether they are published or not. The documents may come from teaching and research institutions in France or abroad, or from public or private research centers.
L'archive ouverte pluridisciplinaire HAL, est destinée au dépôt et à la diffusion de documents scientifiques de niveau recherche, publiés ou non, émanant des établissements d'enseignement et de recherche français ou étrangers, des laboratoires publics ou privés. 


\title{
A thermodynamical approach of contact wear as application of moving discontinuities
}

Received: date/ Accepted: date

\begin{abstract}
The propagation of moving surface inside a body is analysed within the framework of thermomechanical couplings, when the moving surface is associated with an irreversible change of mechanical properties. The moving surface is a surface of heat sources and of entropy production, intensities of which are related to particular energy release rates defined in terms of Hamiltonian gradients. As example, we analyse wear process. Wear phenomena due to contact and relative motion between two solids depend on the loading conditions and material mechanical properties. Friction between contacting bodies induces damage of materials, producing surface and subsurface cracks. Particles are detached from sound solids when some local criteria are satisfied at the boundary. By the way as wears occurs, geometrical changes take place and contact conditions are modified, the particle induce a specific layer with particular properties. Then the interface between the bodies is a complex medium made of detached particles, eventually a lubricant fluid, and damaged zones.We propose to describe the evolution of the interface using the framework developped before for inducing the general form of a wear-law.
\end{abstract}

\section{Introduction}

In the recent past, the propagation of damage has been studied in connection with fracture mechanics and different approaches based on macroscopic or microscopic descriptions of mechanical degradation properties have been proposed.

During a loading history damage in continuum mechanics can be induced by the initiation and the growth of micro-cracks and micro-cavities. These descriptions, which are based on the evolution of the microscopic properties, propose to take the growth of pores or micro-cracks into account, through the idea that when some threshold value is reached, the material cannot support further tensile loading.

Variational formulations were performed to describe the evolution of the surface between the sound and damaged material [3][9]. In the framework of thermomechanical coupling as in fracture mechanics the analysis defines two different energy release rates associated with heat production and entropy production. [10].

This paper is concerned mostly with the description of damage involved on the evolution of a moving interface along which mechanical transformation occurs. Some connections can be made with the notion of configurational forces, [6][8][11][5].

C. Stolz

CNRS UMR7649, Laboratoire de Mécanique des Solides,

Ecole Polytechnique, 91128 Palaiseau France

E-mail: stolz@lms.polytechnique.fr 


\section{General features.}

The domain $\Omega$ is composed of two distinct volumes $\Omega_{1}, \Omega_{2}$ of two materials with different mechanical characteristics. The bounding between the two phases is perfect and the interface is denoted by $\Gamma$, $\left(\Gamma=\partial \Omega_{1} \cap \partial \Omega_{2}\right.$ ). The external surface $\partial \Omega$ is decomposed in two parts $\partial \Omega_{\mathrm{u}}$ and $\partial \Omega_{\mathrm{T}}$ on which the displacement $\mathbf{u}^{d}$ and the loading $\mathbf{T}^{d}$ are prescribed respectively.

The material 1 changes into material 2 along the interface $\Gamma$ by an irreversible process. Hence $\Gamma$ moves with the normal velocity $\mathbf{c}=\phi \boldsymbol{\nu}$ in the reference state, $\boldsymbol{\nu}$ is the outward $\Omega_{2}$ normal, then $\phi$ is positive.

When the surface $\Gamma$ is moving, all the mechanical quantities $f$ can have a jump denoted by $[f]_{\Gamma}=$ $f_{1}-f_{2}$, and any volume average has a rate defined by

$$
\frac{\mathrm{d}}{\mathrm{d} t} \int_{\Omega(\Gamma)} f \mathrm{~d} \Omega=\int_{\Omega(\Gamma)} \dot{f} \mathrm{~d} \Omega-\int_{\Gamma}[f]_{\Gamma} c . \nu \mathrm{d} S
$$

The state of the system is characterized by the displacement field $\mathbf{u}$, from which the strain field $\varepsilon$ is derived. The other parameters are the temperature $T$ and the spatial distribution of the two phases given by the position of the boundary $\Gamma$.

We analyse quasistatic evolution of $\Gamma$ under given loading and displacements prescribed on the boundary $\partial \Omega$.

The behaviour of the phase $i$ is defined by the free energy density $w_{i}$, function of the strain $\varepsilon$ and of the temperature $T$. The mass density $\rho_{i}$ of each phase is the same $\left(\rho=\rho_{i}\right)$. The state equations of each phase are

$$
\sigma_{i}=\rho \frac{\partial w_{i}}{\partial \varepsilon}, \quad s_{i}=-\frac{\partial w_{i}}{\partial T},
$$

where $\sigma_{i}$ is the reversible stress and $s_{i}$ the entropy. If the materials have no viscosity then $\sigma_{i}$ is the stress satisfying the momentum equation.

Assume now that the two phases are linear elastic materials and that the temperature is fixed.

The two phases are linear elastic. The potential energy $\mathcal{E}$ of the structure $\Omega\left(\Omega_{1} \cup \Omega_{2}\right)$ has the following form

$$
\mathcal{E}\left(\mathbf{u}, T, \Gamma, \mathbf{T}^{d}\right)=\sum_{i=1,2} \int_{\Omega_{i}} \rho w_{i}(\varepsilon(u), T) \mathrm{d} \Omega-\int_{\partial \Omega_{\mathrm{T}}} \mathbf{T}^{d} \cdot \mathbf{u} \mathrm{d} S,
$$

where $\partial \Omega_{\mathrm{T}} \cup \partial \Omega_{\mathrm{u}}=\partial \Omega$. The potential energy plays the role of the global free energy in a thermodynamical description ; we can notice that the position of the interface $\Gamma$ becomes an internal parameter for the global system. The characterization of an equilibrium state is given by the stationarity of the potential energy

$$
\frac{\partial \mathcal{E}}{\partial \mathbf{u}} \cdot \delta \mathbf{u}=\sum_{i=1,2} \int_{\Omega_{i}} \rho \frac{\partial w_{i}}{\partial \varepsilon}: \varepsilon(\delta \mathbf{u}) \mathrm{d} \Omega-\int_{\partial \Omega_{\mathrm{T}}} \mathbf{T}^{d} . \delta \mathbf{u} \mathrm{d} S=0
$$

for all $\delta \mathbf{u}$ kinematically admissible field satsfying $\delta \mathbf{u}=0$ over $\partial \Omega_{\mathrm{u}}$. This formulation is equivalent to the set of local equations :

- local constitutive relations:

$$
\sigma_{i}=\rho \frac{\partial w_{i}}{\partial \varepsilon}=\mathbb{C}_{i}: \varepsilon, \text { on } \Omega_{i}
$$

- momentum equations

$$
\operatorname{div} \sigma_{i}=0, \text { on } \Omega_{i},[\sigma]_{\Gamma} . \nu=0 \text { over } \Gamma, \sigma \cdot \mathbf{n}=\mathbf{T}^{d} \text { over } \partial \Omega_{\mathrm{T}},
$$

- compatibility relations

$$
2 \varepsilon=\nabla \mathbf{u}+\nabla^{t} \mathbf{u},[\mathbf{u}]_{\Gamma}=0 \text { over } \Gamma, \mathbf{u}=\mathbf{u}^{d} \text { over } \partial \Omega_{\mathrm{u}} .
$$


They are equations of a problem of heterogeneous elasticity. The solution is denoted by $\mathbf{u}^{\text {sol }}$, this field depends upon the quantities $\left(u^{d}, \mathbf{T}^{d}, \Gamma\right)$. For an equilibrium state

$$
\mathcal{E}\left(\mathbf{u}^{\text {sol }}, \mathbf{T}^{d}, \Gamma\right)=W\left(\mathbf{u}^{d}, \mathbf{T}^{d}, \Gamma\right) .
$$

This equation expresses the fact that the position of the interface $\Gamma$ plays the role of internal parameters.

At a given state of equilibrium for a given value of the prescribed loading $\left(\mathbf{u}^{d}, \mathbf{T}^{d}\right)$, the position for the interface $\Gamma$ is known. At this time a variation of the loading is imposed, the mechanical quantities evolve and propagation of the interface can occur according to a given evolution law. For a prescribed history of the loading, we must determine the rate of all mechanical fields and the normal propagation $\phi$ to characterize the position of the interface $\Gamma$ at each time. Along interface $\Gamma$ perfect bounding is preserved at each time. Let us introduce the notion of convected derivative.

Convected Derivation : The convected derivative $\mathcal{D}_{\phi}$ of any function $f\left(\mathbf{X}_{\Gamma}, t\right)$ is

$$
\mathcal{D}_{\phi} f=\lim _{\tau \rightarrow 0} \frac{f\left(\mathbf{X}_{\Gamma}+\phi \boldsymbol{\nu} \tau, t+\tau\right)-f\left(\mathbf{X}_{\Gamma}, t\right)}{\tau} .
$$

With this definition, we can express the transport of the normal vector at point $\mathbf{x}$ as

$$
\mathcal{D}_{\phi} \boldsymbol{\nu}=-\nabla \phi \cdot \mathbf{e}_{\alpha} \mathbf{e}_{\alpha}
$$

where $\mathbf{e}_{1}, \mathbf{e}_{2}$ is a basis of the plane tangent to $\Gamma$ at point $\mathbf{x}$. We can notice that the equation of the surface $\Gamma, S(\mathbf{X}, t)=0$ satisfies immediatedly

$$
\mathcal{D}_{\phi} S=\frac{\partial S}{\partial \mathbf{X}} \cdot \dot{\mathbf{X}}+\frac{\partial S}{\partial t}=0
$$

which defines the normal velocity $\mathbf{c}$ of $\Gamma$ :

$$
\mathbf{c}=\phi \boldsymbol{\nu}, \quad \boldsymbol{\nu}=\frac{\partial S}{\partial \mathbf{X}} /\left\|\frac{\partial S}{\partial \mathbf{X}}\right\|
$$

and finally for any differentiable fields $f$ the convected derivative is obvious by

$$
\mathcal{D}_{\phi} f=\frac{\partial f}{\partial t}+\phi \nabla f . \nu \text {. }
$$

Hadamard's relations The bounding being perfect between the phases, the temperature, the displacement and the stress vector are continuous along $\Gamma$. Their rates have discontinuities according to the general compatibility conditions of Hadamard, rewritten in terms of the convected derivative :

- continuity of displacement

$$
[\mathbf{u}]_{\Gamma}=0 \Rightarrow \mathcal{D}_{\phi}\left([\mathbf{u}]_{\Gamma}\right)=[\mathbf{v}]_{\Gamma}+\phi[\nabla \mathbf{u}]_{\Gamma} . \boldsymbol{\nu}=0,
$$

- continuity of the stress vector

$$
[\sigma]_{\Gamma} . \boldsymbol{\nu}=0 \Rightarrow \mathcal{D}_{\phi}\left([\sigma]_{\Gamma} . \boldsymbol{\nu}\right)=[\dot{\sigma}]_{\Gamma} . \boldsymbol{\nu}-\operatorname{div}_{\Gamma}\left([\sigma]_{\Gamma} \phi\right)=0 .
$$

The last equation is obtained taking the equilibrium equation into account. As we have

$$
\mathcal{D}_{\phi}\left([\sigma]_{\Gamma} . \boldsymbol{\nu}\right)=\mathcal{D}_{\phi}[\sigma]_{\Gamma} . \boldsymbol{\nu}+[\sigma]_{\Gamma} \cdot \mathcal{D}_{\phi} \boldsymbol{\nu}
$$

where

$$
\mathcal{D}_{\phi}[\sigma]_{\Gamma}=[\dot{\sigma}]_{\Gamma}+\phi \boldsymbol{\nu} \cdot[\nabla \sigma]_{\Gamma} . \boldsymbol{\nu},
$$

and using the conservation of momentum

$$
\mathbf{e}_{\alpha} \cdot \nabla \sigma \cdot \mathbf{e}_{\alpha}+\boldsymbol{\nu} \cdot \nabla \sigma \cdot \boldsymbol{\nu}=0
$$

and the expression of the surface divergence given by

$$
\operatorname{div}_{\Gamma} F=\operatorname{div} F-\boldsymbol{\nu} . \nabla F . \boldsymbol{\nu},
$$

the above result is obtained. 
Orthogonality property for discontinuities. Since the displacement is continuous along the interface,

$$
[\mathbf{u}]_{\Gamma}=0, \Rightarrow[\nabla \mathbf{u}]_{\Gamma} \cdot \mathbf{e}_{\alpha}=0
$$

the discontinuities of the gradient must satisfy

$$
[\nabla \mathbf{u}]_{\Gamma}=\mathbf{U}(x) \otimes \boldsymbol{\nu}
$$

Since the stress vector is continuous on $\Gamma$,

$$
[\sigma]_{\Gamma} . \nu=0
$$

the discontinuities of $\sigma$ and of $\nabla \mathbf{u}$ have the property of orthogonality as pointed in [7] :

$$
[\sigma]_{\Gamma}:[\nabla \mathbf{u}]_{\Gamma}=0
$$

\section{Dissipation analysis}

The mass conservation leads to the continuity of the mass flux $m=\rho \phi$. The first and the second law of thermodynamics give rise to local equations inside the volume and along the moving boundary $\Gamma$ :

$$
\begin{aligned}
\rho \dot{\mathrm{e}_{i}} & =\sigma_{i}: \dot{\varepsilon}-\operatorname{div} \mathbf{q}, \text { over } \Omega_{i}, \\
0 & =m[\mathrm{e}]_{\Gamma}-\boldsymbol{\nu} \cdot \sigma \cdot[\mathbf{v}]_{\Gamma}+\boldsymbol{\nu} \cdot[\mathbf{q}]_{\Gamma}, \text { on } \Gamma,
\end{aligned}
$$

$\mathrm{e}_{i}$ is the internal energy density $\left(\mathrm{e}_{i}=w_{i}+T s_{i}\right)$, and $\mathbf{q}$ is the heat flux associated to the heat conduction.

Thanks to Hadamard compatibility equations, the heat power supply is given in terms of a release rate of internal energy $\mathcal{G}_{t h}$ as an objective quantity defined along $\Gamma$

$$
-\boldsymbol{\nu} \cdot[\mathbf{q}]_{\Gamma}=\mathcal{G}_{t h} \phi, \text { with } \mathcal{G}_{t h}=\rho[\mathrm{e}]_{\Gamma}-\sigma:[\varepsilon]_{\Gamma} .
$$

The value of $\mathcal{G}_{t h}$ is obtained considering the orthogonality condition on the discontinuities. When $\phi=0$ in the reference state the interface $\Gamma$ does not move, and the normal heat flux is continuous. When the transformation occurs, the moving interface is a surface of heat sources intensities which are given by $\mathcal{G}_{t h} \phi$.

The total internal energy of the structure is

$$
\mathrm{E}\left(\mathbf{u}, \Gamma, T, T^{d}\right)=\int_{\Omega(\Gamma)} \rho \mathrm{e} \mathrm{d} \Omega-\int_{\partial \Omega_{\mathrm{T}}} \mathbf{T}^{d} \cdot \mathbf{u} \mathrm{d} S=\mathcal{E}+\int_{\Omega(\Gamma)} \rho s T \mathrm{~d} \Omega .
$$

For quasistatic evolution, the first law of thermodynamics is written as follows :

$$
\frac{\mathrm{d}}{\mathrm{d} t} \mathrm{E}-\frac{\partial \mathrm{E}}{\partial \mathbf{T}^{d}} \cdot \dot{\mathbf{T}}^{d}=-\int_{\partial \Omega} \mathbf{q} \cdot \mathbf{n} \mathrm{d} S,
$$

and taking into account the conservation of momentum, we have

$$
\frac{\partial \mathrm{E}}{\partial \Gamma} \cdot \dot{\Gamma}=\int_{\Gamma}[\mathbf{q}]_{\Gamma} . \nu \mathrm{d} S=-\int_{\Gamma} \mathcal{G}_{t h} \phi \mathrm{d} S
$$

then the derivative of the total energy relatively with respect to the position of the interface determines the source of heat due to the irreversible process, intensity of which is governed by the internal ernergy release rate:

$$
\mathcal{G}_{t h}=-\frac{\partial \mathrm{E}}{\partial \Gamma}
$$

The entropy production is given by

$$
\int_{\Omega}\left(\rho \dot{s}+\frac{\operatorname{div} \mathbf{q}}{T}-\mathbf{q} \cdot \frac{\nabla T}{T^{2}}\right) \mathrm{d} \Omega+\int_{\Gamma}\left(-m[s]_{\Gamma}-\boldsymbol{\nu} \cdot\left[\frac{\mathbf{q}}{T}\right]_{\Gamma}\right) \mathrm{d} S \geq 0 .
$$


Under the assumption of separability of the two dissipations, the term inside the volume is reduced to the conduction, and the term along the surface is then

$$
D_{\Gamma}=\frac{\rho[w]_{\Gamma}-\sigma:[\varepsilon]_{\Gamma}}{T} \phi=\frac{\mathcal{G}_{s}}{T} \phi \geq 0,
$$

where $\mathcal{G}_{s}$ is the release rate of free energy.

This quantity has an analogous form to the driving traction force acting on a surface of strain discontinuity introduced in [1] . The criteria which guide the evolution of the interface may be written as function of this quantity.

In a thermomechanical coupling, two different release rates must be distinguished. One defined in terms of variation of the total internal energy gives rise to the heat source associated with the moving surface ; the second one gives rise to the production of entropy.

In the case of isothermal evolution the total dissipation is given in terms of the derivative of the potential energy relatively to the position of the interface

$$
\frac{\partial \mathcal{E}}{\partial \Gamma} \cdot \dot{\Gamma}=-\int_{\Gamma} \mathcal{G}_{s} \phi \mathrm{d} S, \text { or } \mathcal{G}_{s}(\mathbf{x})=-\frac{\partial \mathcal{E}}{\partial \Gamma}(\mathbf{x}) .
$$

whith $\mathcal{G}_{s}=\rho[w]_{\Gamma}-\sigma:[\varepsilon]_{\Gamma}$.

In this case, there is only one energy release rate to characterize the propagation, it gives the sources of entropy production and the dissipation.

These relations can be generalized to the dynamical case, by replacing the internal energy of the system by its Hamiltonian, and can be extended to the case of running cracks, and to more general behaviour and structures [10].

\section{Dissipation analysis in dynamical case.}

Now, we take the inertia effects into account. Thus the two thermodynamics principles must be rewritten. The mass conservation leads to the continuity of the mass flux $m=\rho \phi$, where $\rho$ denotes the mass density. The first law and the second law of thermodynamics give rise to local equations inside the volume and along the moving surface $\Gamma$ :

$$
\begin{aligned}
\rho \dot{e}_{i} & =\sigma_{i}: \dot{\varepsilon}-d i v q, \text { in } \Omega_{i}, \\
0 & =m\left[\mathrm{e}+\frac{\mathbf{v}^{2}}{2}\right]_{\Gamma}-\boldsymbol{\nu} \cdot[\sigma \cdot \mathbf{v}]_{\Gamma}+\boldsymbol{\nu} \cdot[\mathbf{q}]_{\Gamma}, \text { on } \Gamma .
\end{aligned}
$$

Then taking the conservation of the momentum and the continuity of the displacement into account :

$$
[\mathbf{u}]_{\Gamma}=0, \quad[\sigma]_{\Gamma} . \nu=m[\mathbf{v}]_{\Gamma} .
$$

we obtain the heat power supply defined by the internal energy release rate $\mathcal{G}_{t h}\left(\bar{\sigma}=\frac{1}{2}\left(\sigma_{1}+\sigma_{2}\right)\right)$ :

$$
-\boldsymbol{\nu} \cdot[\mathbf{q}]_{\Gamma}=\mathcal{G}_{t h} \phi, \quad \mathcal{G}_{t h}=\rho[\mathrm{e}]_{\Gamma}-\boldsymbol{\nu} \cdot \bar{\sigma} \cdot[\nabla \mathbf{u}]_{\Gamma} . \nu .
$$

The Hamiltonian of the structure is the sum of the kinetic energy and the total internal energy, the potential energy is defined as above :

$$
\mathcal{H}=\int_{\Omega} \frac{\mathbf{p}^{2}}{2 \rho} \mathrm{d} \Omega+\mathcal{E}+\int_{\Omega} \rho s T \mathrm{~d} \Omega
$$

The momentum conservation is then defined by the set of equations

$$
\begin{array}{r}
\frac{\partial \mathcal{H}}{\partial \mathbf{p}} \bullet \delta \mathbf{p}=\int_{\Omega} \mathbf{v} . \delta \mathbf{p} \mathrm{d} \Omega \\
\frac{\partial \mathcal{H}}{\partial \mathbf{u}} \bullet \delta \mathbf{u}=-\frac{d}{d t} \int_{\Omega} \mathbf{p} . \delta \mathbf{u} \mathrm{d} \Omega
\end{array}
$$


where $\mathbf{p}$ is the momentum, these equations leads to the classical equation of motion. The first law of thermodynamics is rewritten as follows :

$$
\frac{d \mathcal{H}}{d t}-\frac{\partial \mathcal{H}}{\partial \mathbf{T}^{d}} \cdot \dot{\mathbf{T}}^{d}=\int_{\partial \Omega}-\mathbf{q} \cdot \mathbf{n} \mathrm{d} S,
$$

and taking into account of the momentum conservation, we have

$$
\frac{\partial \mathcal{H}}{\partial \Gamma} \cdot \dot{\Gamma}=\int_{\Gamma}[\mathbf{q}]_{\Gamma} . \nu \mathrm{d} S=-\int_{\Gamma} \mathcal{G}_{t h} \phi \mathrm{d} S
$$

the second law has the same form as previously. The interface is perfect at each time, under the assumption of separability of the two dissipations, the term inside the volume is reduced to the conduction, and the term along the surface is then : $D_{\Gamma}=\frac{\mathcal{G}_{s}}{T} \phi$ where $\mathcal{G}_{s}$ has also the form of a release rate of energy.

$$
\mathcal{G}_{s}=\rho[w]_{\Gamma}-\boldsymbol{\nu} \cdot \bar{\sigma} \cdot[\nabla \mathbf{u}]_{\Gamma} . \nu .
$$

In a thermomechanical coupling, two different release rates must be distinguish, one defined in term of variation of the Hamiltonian give rise the heat source associated with the moving surface, the second one describes the production of entropy.

In the case of isothermal evolution, we can define another Hamiltonian

$$
\mathcal{H}=\int_{\Omega} \frac{\mathbf{p}^{2}}{2 \rho} \mathrm{d} \Omega+\mathcal{E},
$$

and the total dissipation is then given by :

$$
\frac{\mathrm{d}}{\mathrm{d} t} \mathcal{H}-\frac{\partial \mathcal{H}}{\partial \mathbf{T}^{d}} \cdot \dot{\mathbf{T}}^{d}=\frac{\partial \mathcal{H}}{\partial \Gamma} \cdot \dot{\Gamma}=-\int_{\Gamma} \mathcal{G}_{d y n} \phi \mathrm{d} S,
$$

where $\mathcal{G}_{d y n}=\rho[w]_{\Gamma}-\boldsymbol{\nu} \cdot \bar{\sigma} \cdot[\nabla \mathbf{u}]_{\Gamma} . \nu$.

\section{A thermodynamical approach to contact wear}

The system consists in two sliding contacting bodies $\Omega_{1}, \Omega_{2}$ separated by a contact interface $\Omega_{3}$. We assume the properties of $\Omega_{3}$ are known, and we attemp to characterized the behaviour and the evolution of the interface, taking into account modelization of wear phenomena. Such an interface $\Omega_{3}$ must be

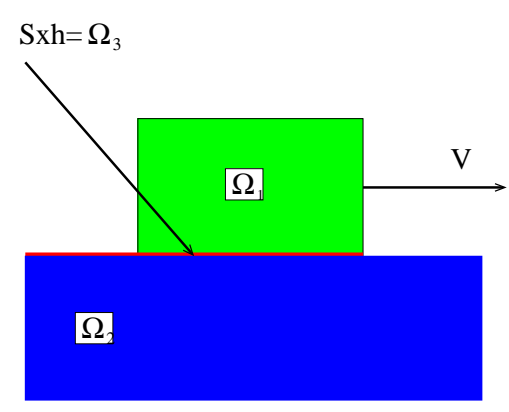

Fig. 1 Macroscopic description of contact.

considered at a macroscopic level as an homogeneous body obtained by some averaging process through the thickness $H$ of $\Omega_{3}$. This thickness is so small compared with the size of the contact zone and of the tribological system that the condition of homogeneity can be useful.

The behaviour of each zone is defined through a free energy $w_{i}$ and a dissipation potential. As a result of wear, the boundary $\Gamma_{i}$ moves. Along each front $\Gamma_{i}$, the normal $\boldsymbol{\nu}_{i}$ is oriented toward the sound 


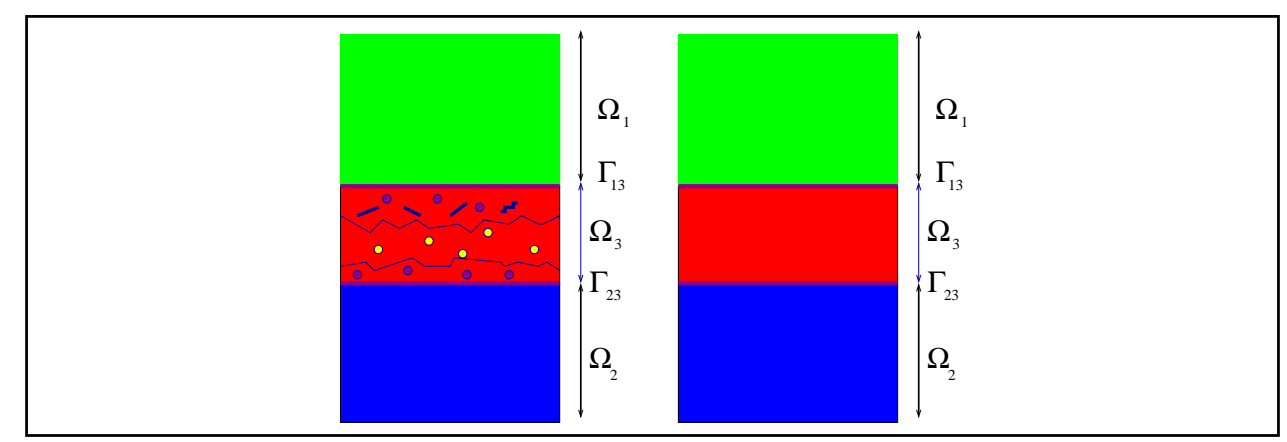

Fig. 2 The moving boundaries and the interface medium.

solid. We denote by $\phi_{i} \boldsymbol{\nu}_{i}$ the normal velocity of the surface $\Gamma_{i}$. Along these boundaries, mechanical quantities avec jump $[f]_{\Gamma_{i}}=f_{i}^{+}-f_{i}^{-}$. By expressing the conservation laws in $\Omega_{i}$ and accross each $\Gamma_{i}$, we get a set of local equations for the characterisation of the rate quantities :

- mass conservation

$$
\left\{\begin{array}{cl}
\text { on } \Gamma_{i}, & m_{i}=\rho_{i} \phi_{i} \boldsymbol{\nu}_{i} \\
\text { in } \Omega_{i}, & \dot{\rho}_{i}+\operatorname{div} \rho_{i} \mathbf{v}=0
\end{array}\right.
$$

- momentum conservation

$$
\left\{\begin{array}{cc}
\text { on } \Gamma_{i}, & \sigma . \nu_{i}=0 \\
\text { in } \Omega_{i}, & \operatorname{div} \sigma=0
\end{array}\right.
$$

- energy balance equation

$$
\left\{\begin{array}{cl}
\text { on } \Gamma_{i}, & m_{i}[w+s T]_{\Gamma_{i}}-\boldsymbol{\nu}_{i} \sigma \cdot[\mathbf{v}]_{\Gamma_{i}}+\boldsymbol{\nu}_{i} \cdot[\mathbf{q}]_{\Gamma_{i}}=0 \\
\text { in } \Omega_{i}, & \rho \dot{\mathrm{e}}=\sigma: \varepsilon(\mathbf{v})-\operatorname{div} \mathbf{q} .
\end{array}\right.
$$

- continuity of the displacement along each $\Gamma_{i}$

$$
[\mathbf{u}]_{\Gamma_{i}}=0 \Rightarrow[\mathbf{v}]_{\Gamma_{i}}+\phi_{i}[\nabla \mathbf{u}]_{\Gamma_{i}} . \boldsymbol{\nu}_{i}=0
$$

\section{The dissipation}

The internal entropy production is positive and is decomposed in three contributions :

- the volume thermal conduction

$$
D_{t h}=-\mathbf{q} \cdot \frac{\nabla T}{T^{2}}
$$

- the volume term due to intrinsic mechanical irreversibility

$$
D_{m}=\frac{1}{T}(\sigma: \operatorname{grad} \mathbf{v}-\rho(\dot{w}+s \dot{T})),
$$

- the surface term due to mechanical discontinuities

$$
D_{\Gamma}=\frac{1}{T}\left(m_{i}[w]_{\Gamma_{i}}-\boldsymbol{\nu}_{i} \cdot \sigma \cdot[\mathbf{v}]_{\Gamma_{i}}\right)
$$

We can notice that if the mass flux $m_{i}$ is zero, the velocity $\phi_{i}$ is zero too. Then the velocity jump verifying Hadamard relation's is zero, and no dissipation along $\Gamma_{i}$ occurs. The dissipation $D_{\Gamma}$ is then a characteristic of loss of matter and so of the phenomenon of wear. 
Description of the interface The interface $\Omega_{3}$ is described by it's middle surface $\Gamma$ with equation $S(X, t)=0$, normal vector $\mathbf{n}(X, t)$ and thickness $H(X, t)=2 h(X, t)$.

The two boundaries $\Gamma_{13}, \Gamma_{23}$ are then defined by

$$
x_{1}=X+h(X, t) \mathbf{n}, \quad x_{2}=X-h(X, t) \mathbf{n} .
$$

Denoting $\phi$ the velocity of $\Gamma$, it is obvious that

$$
\mathcal{D}_{\phi}\left(x_{1}\right)=\phi_{1} \boldsymbol{\nu}_{1}, \quad \mathcal{D}_{\phi}\left(x_{2}\right)=\phi_{2} \boldsymbol{\nu}_{2} .
$$

The continuity of the displacement is then rewritten as

$$
[\mathbf{u}(X \pm h(X, t) \mathbf{n}, t)]_{\Gamma_{i}}=0 .
$$

The dissipation by unit area of contact surface along $\Gamma$ is then

$$
D_{m}=D_{\Gamma_{13}} j_{1}+D_{\Gamma_{23}} j_{2}+\int_{H} d_{m} j(z) d z,
$$

where $j_{1}=j(h), j_{2}=j(-h), j(z)=\operatorname{det}(\mathrm{I}-z \mathrm{~b})$ and $\mathrm{b}$ is the curvature tensor.

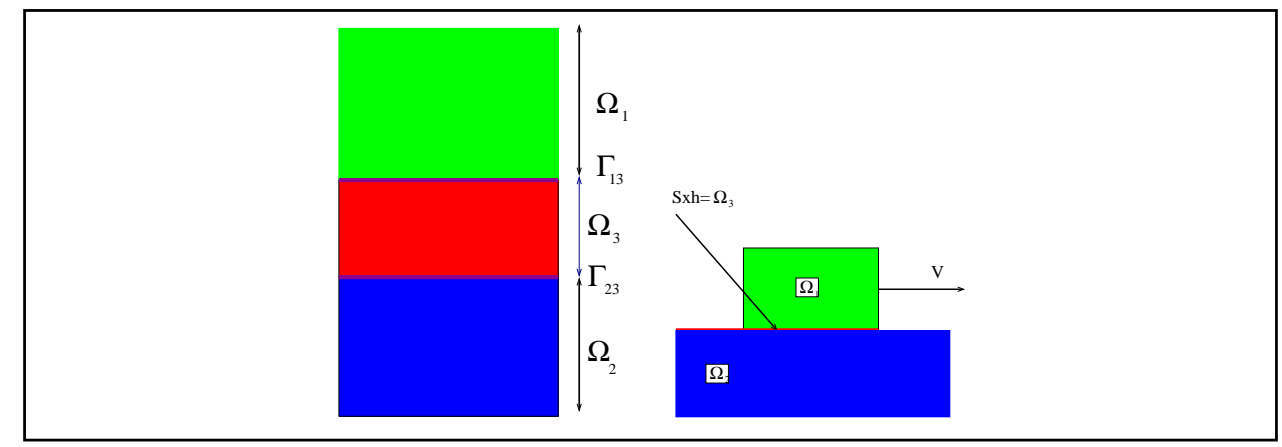

Fig. 3 The mesoscopic description.

Interpretation The contributions on the dissipation have different nature. The dissipation along the interface $\Gamma_{13}, \Gamma_{23}$ are characteristics of the loss of sound material. The last term is the dissipation due to the irreversibility inside the interface. For example, if $\Omega_{3}$ is a viscous fluid, this term is due to the shear stresses, and consequently we have the possibility to describe some resistance to glide. The term $D_{3}$

$$
D_{3}=\int_{h} d_{m} j(z) d z=\int_{H}(\sigma: \dot{\varepsilon}-\dot{w}) j(z) d z,
$$

is interpreted in order to describe the friction associated with the relative motion of solids.

For a given mechanical behaviour of the interface, the resolution of the evolution equation must be completed by evolution laws in order to determine the propagation of the damage material. For example, we consider a Griffith's criterion :

$$
\begin{cases}\mathcal{G}(X, t)<G_{c}, & \phi=0, \\ \mathcal{G}(X, t)=G_{c}, & \phi \geq 0 .\end{cases}
$$

The main difficulty is to choose a good modelization for the behaviour in the interface and a procedure for its own identification. 


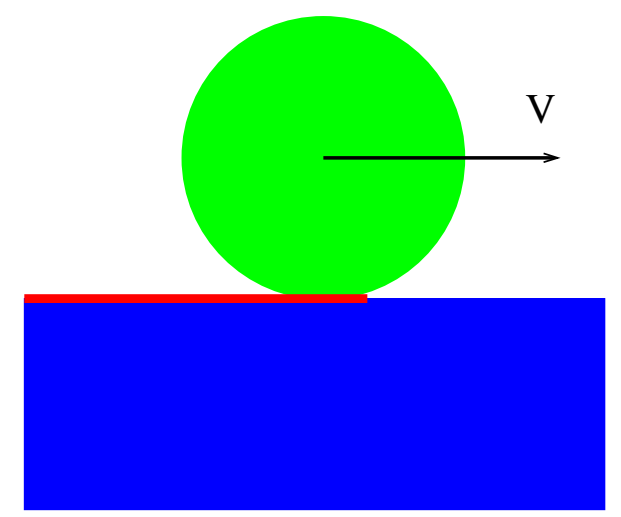

Fig. 4 A moving punch on an elastic half-space.

\section{An example}

Consider now an example of such a behaviour, to emphasize the possibility to describe the friction as well as the wear phenomena in the same approach. Consider a rigid punch $\Omega_{1}$ moving on a half elastic plane. The interface is composed by a viscous fluid with particles in suspension, the global behaviour of this fluid is defined by a bulk modulus $k$ and a viscous modulus $\eta$ which are functions of the concentration of the particles. This particular case has been studied using of integral equations [4].

We consider that the shear is essentially due to viscosity and the elastic behaviour has only uniaxial effect in the direction $\mathbf{e}_{y}$. The motion inside $\Omega_{3}$ is approximated by a linear profile for the velocities and displacement with respect to the normal coordinate of the middle surface $S$. Then $\dot{\varepsilon}$ is associated with the jump of the velocities.

Then the constitutive laws inside $\Omega_{3}$ are given by :

$$
\sigma_{x y}=m(c)\left(\dot{u}_{x}^{1}-\dot{u}_{x}^{2}\right), \quad \sigma_{y y}=k(c)\left(u_{y}^{1}-u_{y}^{2}\right) .
$$

These expressions are compatible with experimental observations. We are interested now on a steadystate solution, then

$$
\dot{u}_{x}^{1}-\dot{u}_{x}^{2}=-V\left(\dot{u}_{x, x}^{1}-\dot{u}_{x, x}^{2}\right) .
$$

The half plane has an elastic linear behaviour, the displacement on the interface $\Gamma_{23}$ is given by solving the Galin's Equations :

$$
\begin{aligned}
& c o_{1} u_{x, x}(x)=c o_{2} \sigma_{y y}(x)+V p \frac{1}{\pi} \int_{-a}^{a} \frac{\sigma_{x y}(s)}{s-x} d s, \\
& c o_{1} u_{y, x}(x)=-c o_{2} \sigma_{x y}(x)+V p \frac{1}{\pi} \int_{-a}^{a} \frac{\sigma_{y y}(s)}{s-x} d s,
\end{aligned}
$$

where the coefficients are :

$$
c o_{1}=\frac{E}{2\left(1-\nu^{2}\right)}, \quad c o_{2}=\frac{(1-2 \nu)}{2(1-\nu)},
$$

and $E$ is the Young's modulus, $\nu$ is the Poisson's ratio, $V p f$ is the principal value of $f$ in the sense of Cauchy.

The solution is founded by a method of perturbation, using an asymptotic expansion with respect to the concentration $c$ of particles. Finally we get the following results :

- at the 0 order, the Hertz contact solution is recovered,

- at the first order, a dependance with the concentration is obtained. This depends upon the criterion of wear. For sake of simplicity a linear law is chosen, the velocity of progagation is given by $\phi=\lambda \sigma_{y y}^{2}$. 


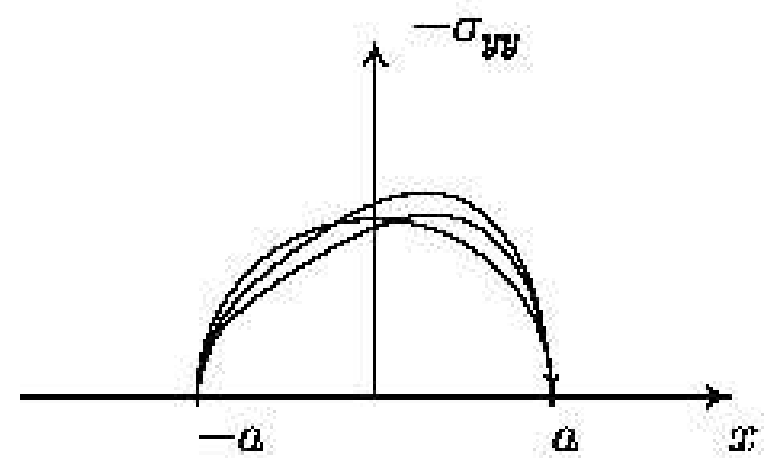

Fig. 5 Pressure in the contact area.

Due to the presence of a viscous fluid, the maximum pressure under the punch is not in the center of the contact area, as in the case of contact with friction, [2]. This example shows the pertinence of the modelization and the possibility of studying a problem of thin layer interface for describing the interaction between wear and friction.

Global approach of the interface With the same hypotheses, we take the average value of the free energy to define the global free energy of the interface per unit of contact area.

$$
\psi_{S}\left(\mathbf{u}_{1}, \mathbf{u}_{2}, \alpha\right)=\frac{1}{\rho_{S}} \int_{H} \rho(x, z) w_{3}(\varepsilon(x+z \mathbf{n}), \alpha) d z
$$

where $\alpha$ denotes internal parameters, and $\rho_{S}$ is the mass density defined by area unit too :

$$
\rho_{S}=\int_{H} \rho(x, z) d z
$$

In the same way, we can define the potential of dissipation

$$
\mathrm{D}_{S}\left(\mathbf{v}_{1}, \mathbf{v}_{2}, \dot{\alpha}\right)=\frac{1}{\rho_{S}} \int_{H} \rho(x, z) \mathrm{d}_{3}(\dot{\varepsilon}(x+z \mathbf{n}), \dot{\alpha}) d z
$$

As pointed out, the equilibrium along the interface is defined by the equations obtained by variations of the potential energy with respect to the fields $\mathbf{u}_{1}, \mathbf{u}_{2}$. Then we obtain :

$$
\sigma . \mathbf{n}_{i}=\rho_{S}\left(\frac{\partial \psi_{S}}{\partial \mathbf{u}_{i}}+\frac{\partial \mathrm{D}_{S}}{\partial \mathbf{v}_{i}}\right)
$$

This result suggests a more general study of the behaviour of the interface by asymptotic expansion of the displacement

$$
\mathbf{u}_{3}=\mathbf{u}_{o}+z \mathbf{u}_{1}+z^{2} \mathbf{u}_{2}+\ldots
$$

with respect to the normal coordinate inside the interface $\Omega_{3}$. Then specific functions for the constitutive behaviour should be deduced in the same manner. 


\section{Conclusion}

We have developped a thermodynamical approach of contact wear based on moving interface description. In a such description, we emphasize that the wear is governed by local quantities. The dissipation is a function of a local energy release rate. Finally, in a global description we can consider the interface as a thin layer with specific properties. This allows us to take into account of modification of the properties of this interface with the loss of matter due to wear. The main difficulties are now to indentify with macroscopic experimental data such a complex behaviour depending on the degree of refinement of the description.

\section{References}

1. Abeyratne, R., Knowles, J. (1990) On the driving traction acting on a surface of strain discontinuity in a continuum. J. Mech. Phys. Solids, 38(3), 345-360.

2. Bui H.D., Dang Van K. (1976) Contribution 'a l'étude théorique du contact élastique d'un frotteur cylindrique glissant sur un massif élastique. Industrie minérale, No Spécial rhéologie, Tome IV, no 1, pp 1-8.

3. Bui H.D., Dang Van K., Stolz, C. (1981), Formulations variationnelles du problème en vitesse pour le solide élastique fragile avec zones endommagées. C. R. Acad. Sci. Paris, 292(II), 251-254.

4. Dragon-Louiset M. (2001) On a predictive macroscopic contact-sliding wear model based on micromechanical considerations. Int. J. Solids and Structures, 38(9), 1625-1639.

5. Grinfeld, M. (1990) Thermodynamic equilibrium conditions of the phases of a nonlinearly elastic material. Dokl. Akad. Nauti. SSSR, 251, 1-15.

6. Gurtin, M. E. (1995) The nature of configurational forces. Arch. Rat. Mech. Anal., 131, 67-100.

7. Hill, R. (1986) Energy momentum tensors in elastostatics : some reflections on the general theory, J. Mech. Phys. Solids,34(3)305-317.

8. Maugin, G. A. (1995) Material forces : concept and applications. ASME, Appl. Mech. Rev., 48, 213-245.

9. Pradeilles-Duval, R.M., Stolz, C. (1995) Mechanical transformations and discontinuities along a moving surface. J. Mech. Phys. Solids, 43(1), 91-121.

10. Stolz, C. (1995), Functional approach in non linear dynamics, Arch. Mech, 47(3), 421-435

11. Truskinovsky, L.M. (1987) Dynamics of non equilibrium phases boundaries in a heat conducting non linearly elastic medium. P.M.M., 51, 777-784. 